\title{
Los estereotipos de género y las TICs en estudiantes de secundaria
}

\section{Gender stereotype and ICTs in high school students}

\author{
Josefa Castro, Xoán Trigo \\ Universidad de Santiago de Compostela
}

\begin{abstract}
Resumen
Este trabajo explora las diferencias de género en estudiantes de secundaria a la hora de interactuar con las TICs. La investigación fue realizada con una muestra de 351 alumnos de centros educativos públicos de Galicia. Se aplicó un cuestionario en el que se medían aspectos relacionados con el acceso, experiencia, frecuencia y uso que hacían, tanto de los computadores como de internet. Se aplicaron escalas para evaluar la percepción de autoeficacia en el manejo de internet y de computadores, las actitudes hacia los mismos, y los estereotipos de género. Los resultados indican diferencias entre hombres y mujeres en el uso que realizan de internet, más orientado a la comunicación en las mujeres y al entretenimiento en los hombres, así como una mayor tendencia en los hombres a percibir de forma más estereotipada la informática, viéndola como parte del dominio masculino.
\end{abstract}

Palabras-clave: Informática, internet, autoeficacia, estereotipo de género.

\begin{abstract}
This study explored gender differences in high school students when it comes to interacting with ICTs. The research was carried out with a sample of 351 students from public schools in Galicia (Spain). A questionnaire was applied in which measured aspects related to access, experience, frequency and use that students made, both of computers and the Internet. Scales were applied to evaluate computer self-efficacy, internet self-efficacy, attitudes towards the use of ICTs, and gender stereotypes. The results indicated differences between men and women in the use they make of Internet, more oriented to communication in women and more oriented to entertainment in men, as well as a greater tendency in men to perceive the computing more stereotyped, seeing it as part of domain male.

Keywords: Computer use, internet use, self-efficacy, gender stereotype
\end{abstract}

La vertiginosa expansión de internet y la aparición de nuevos dispositivos electrónicos ha trasformado cualitativamente nuestro mundo, hablándose ya de una nueva era. Ser capaz de dominar las nuevas tecnologías es tan importante que los países de nuestro entorno llevan ya una década incorporando las TICs en el curriculum escolar, y considerándolas como una de las competencias básicas de la escolarización obligatoria.

El dominio de las TICs, no solo no se distribuye equitativamente entre países ricos y pobres, sino que también hay diferencias entre hombres y mujeres. En los países más desarrollados tecnológicamente, la llamada brecha digital de género tiende a ir desapareciendo, si bien todavía persisten desequilibrios, y las mujeres siguen siendo una minoría en los estudios y profesiones especializadas en este campo (menos del $20 \%$ de los estudiantes de ingeniería informática o computacional son mujeres).

Las diferencias de género en el dominio de las TICs son un tema ampliamente analizado, especialmente en los últimos años. Hasta no hace mucho tiempo, numerosas investigaciones indicaban que los hombres comenzaban a familiarizarse antes con la informática, y usaban los ordenadores y se conectaban a internet de forma más frecuente que las mujeres (Acosta, Borges, González, Hernández y Rodríguez, 2003; Durndell y Haag, 2002). También presentaban actitudes más positivas hacia la informática y una mayor percepción de autoeficacia computacional (Durndell y Haag, 2002; Li e Kirkup, 2007; Simsek, 2011; Vekiri y Chronaki, 2008). La autoeficacia computacional hace referencia a la confianza que tenemos en nuestras habilidades para realizar tareas relacionadas con un computador, y que incluyen también el dominio de la navegación por internet. Estudios más recientes ofrecen más divergencia en sus resultados, algunas investigaciones no encuentran diferencias entre hombres y mujeres en su percepción de autoeficacia informática (Beierlein, Imhof y Vollmeyer, 2006; Li y Ranieri, 2013), y hay trabajos que indican que son las mujeres las que se perciben más eficaces, en algunas tareas relacionadas con el dominio de internet (Glassman e Kim, 2013; Tomte e Hatlevik, 2011).

Las diferencias entre hombres y mujeres en la percepción de autoeficacia informática parecen deberse a los estereotipos de género, como concluye Whitley (1997) en el metaanálisis que realiza de 95 estudios realizados con muestras norteamericanas. Los hombres comparados con las mujeres, veían los ordenadores como más apropiados para ellos mismos, y se veían a si mismos como más competentes en tareas relacionadas con la informática, además de mostrar actitudes más positivas cara a los computadores. Resultados de estudios posteriores inciden en la persistencia de esa visión estereotipada de la informática, percibiéndose como parte del dominio masculino (Hergatt, Whetten y Huffman, 2013; Li y Kirkup, 2007; Papastergiou, 2008). 
El objetivo de este trabajo es analizar si existen diferencias entre hombres y mujeres en el acceso y frecuencia de uso de los ordenadores y de internet, así como en los tipos de actividades que realizan con ellos. También se analizan diferencias de género en la percepción de la autoeficacia informática, las actitudes y los estereotipos de genero.

\section{Método}

\section{Participantes}

La muestra está formada por 351 alumnos que cursaban desde $2^{\circ}$ curso de la E.S.O. hasta $2^{\circ}$ curso de bachillerato, en seis centros públicos de Galicia (rurales y urbanos, de costa y de interior). Del total de la muestra, $190(54,1 \%)$ son mujeres y $161(45,9 \%)$ son hombres. Las edades oscilaban entre los 13 y los 21 años $(M=15,62, \mathrm{DT}=1,57)$.

\section{Instrumentos}

Se elaboró un cuestionario que permitiese medir todas las variables del estudio en poco tiempo. Los ítems fueron elaborados a partir del análisis de otros instrumentos empleados en la literatura sobre el tema, y se compone de un total de 117 ítems repartidos en tres partes. La primera parte recoge datos demográficos e información sobre las posibilidades de acceso, la experiencia previa, la frecuencia de uso, y el tipo de actividades que hacen, tanto con el ordenador como en internet. La segunda parte recoge información sobre la percepción de autoeficacia en el manejo de computadores y de internet, para lo cual se administró la escala Self Efficacy Internet Computer Task (SEICT) de Tomte y Hatlevik (2011). Esta escala se compone de 14 ítems con un formato de respuesta tipo Likert de 7 puntos. Los 8 primeros ítems miden la autoeficacia en relación al manejo de software de computadoras ("confío en mi capacidad para editar fotografías digitales u otras imágenes gráficas"), y los otros 6 ítems miden la autoeficacia en el uso de internet ("confió en mi capacidad para descargar archivos o programas"). La fiabilidad de esta escala oscila, en los estudios que la emplearon, entre .76 y .86 .

La tercera parte recoge información sobre las actitudes positivas o negativas en relación a los ordenadores y a internet, así como las creencias en los estereotipos de género en relación a este dominio. Para su evaluación se emplearon las escalas: Computer attitude Scale (CAS), de Nickell y Pinto (1986), que consta de 20 ítems que miden, en una escala Likert de 5 puntos (1-5) el grado de acuerdo con frases que muestran creencias positivas y negativas sobre los ordenadores; y la Escala de Estereotipo de Rol Sexual (EERS), compuesta por 5 ítems que contienen frases sobre estereotipos sexuales ("los hombres tienen más habilidades para el uso de ordenadores y para la informática que las mujeres") y que deben valorar su grado de acuerdo en una escala de respuesta tipo Likert de 5 puntos.

\section{Procedimiento}

Los datos fueron recogidos en horario lectivo por uno de los investigadores, explicando la forma de cubrirlos y aclarando cualquier duda que surgiese. Las sesiones duraban entre 15-20 minutos. Los cuestionarios se administraron siguiendo las normas de confidencialidad y consentimiento informado indicadas en el código ético.

\section{Análisis}

Los análisis se realizaron con el paquete estadístico IBM SPSS versión 20. En primer lugar, se llevó a cabo un análisis alfa de Cronbach para comprobar la fiabilidad de los instrumentos empleados. El análisis de la consistencia interna de las escalas mostró una fiabilidad adecuada para la escala de autoeficacia SEICT $(\alpha=.82)$ y para sus dos subescalas: autoeficacia computador $(\alpha=.76)$ y autoeficacia internet $(\alpha=.80)$, así como para la escala de estereotipo de género $(\alpha=.80)$; y algo más baja para la escala de actitudes CAS $(\alpha=.66)$. En segundo lugar, para comprobar la asociación entre sexo y variables sobre el acceso, frecuencia y uso de los ordenadores y de internet aplicamos las pruebas de contraste Chi-cuadrado para tablas de contingencia, U de Mann-Whitney y t de Student en función del tipo de medida de las variables. Por último, se llevó a cabo un análisis de correlación de Pearson para conocer si existía relación entre las puntuaciones de las distintas variables.

\section{Resultados}

En primer lugar analizamos las variables: posibilidades de acceso, experiencia previa, frecuencia, y tipo de actividades que se hacen tanto con los ordenadores como con internet.

Prácticamente todos los sujetos tienen en su casa acceso a internet $(93,4 \%)$ y disponen de uno o varios dispositivos con los que acceder. El 71,5\% dispone de un ordenador fijo, que para cerca de la mitad (47\%) es de su propiedad. Lo más común es que tengan un portátil $(82,3 \%)$ que suele ser propio $(73,3 \%)$, y/o una Tablet $(54,1 \%)$, que también es suya para el $60 \%$. Además, el $98 \%$ dispone de un móvil propio y con acceso a internet $(90 \%)$. Solamente 4 sujetos de nuestra muestra no disponían de ordenador en casa ni de ningún otro dispositivo con el que acceder a internet, incluido un móvil. Tanto chicos como chicas acceden a internet principalmente desde casa, seguido de wifis gratuitas, casas de amigos o familiares, y desde el colegio. Las mujeres hacen más uso de las wifis gratuitas que los hombres $\left(71,2 \%\right.$ vs $58,2 \% ; \chi^{2}=5,254$; g.l. $\left.=1, p<.055\right)$ y acceden más desde la casa de amigos o familiares que los hombres $\left(73,6 \%\right.$ vs $56,6 \% ; X^{2}=9,265 ;$ g.1. $\left.=1, p<.01\right)$.

En relación a la experiencia, más de la mitad $(55 \%)$ han realizado entre 1 o 2 cursos de informática, y afirman llevar más de 5 años usando un ordenador de forma habitual (70) \% y también, más de 5 años accediendo a internet $(65 \%)$. Tanto hombres como mujeres aprendieron sus habilidades computacionales, en primer lugar, a base de experimentar por su cuenta, aunque en mayor medida las chicas manifiestan ser 
autodidactas $\left(77 \%\right.$ vs $65 \% ; \chi^{2}=5,897 ;$ g.1.=1, $\left.p<.05\right)$, seguido de la realización de cursos de informática (45,8\% hombres, 36,5\% mujeres), clases del colegio, también en mayor medida las chicas $(38,9 \%$ vs $24,8 \%$; $\chi^{2}=6.668 ;$ g.l. $=1, p<.01$ ), o con los amigos (30\% las mujeres, $24,8 \%$ los hombres).

La frecuencia en el uso de estas tecnologías es de una media de 1,45 horas al día dedicado al ordenador, y de ese tiempo, prácticamente están conectados siempre a internet (1,37 horas al día). Aplicamos la prueba U de Mann-Whitney para comprobar si había diferencias de género en la frecuencia de empleo del ordenador para realizar tareas escolares y para actividades de ocio o entretenimiento personal. Encontramos un efecto significativo de género en el caso de la frecuencia de uso del ordenador para tareas escolares (U-MannWhitney=6719; $Z=-4,922 ; p<.001)$. Concretamente, el ordenador es empleado con fines escolares significativamente con mayor frecuencia por las mujeres.

Finalmente les preguntábamos la frecuencia con que usaban los ordenadores o accedían a internet para distintas actividades. Como podemos observar (tabla 3) hay diferencias de género en las actividades que suelen realizar. Los hombres pasan más tiempo que las mujeres con los juegos online $(M=2.16, D T=1.36 ; t=-9.282$; g.l. $=349, \quad p<.001)$, participando en foros $(M=0,70$, $D T=.84 ; t=-2.303 ; p<0.5)$ y descargando programas $(M=1.94, D T=1 ; \mathrm{t}=-1.723 ; p<.01) ; \mathrm{y}$ las mujeres usan más internet para conectarse a redes sociales $(M=3.05$, $D T=1.19 ; t=3.888 ; p<.001)$, chatear $(M=2.77, D T=1,27$; $t=2.701 ; p<.01)$, enviar e-mails $(M=2.04, D T=1.04)$; $t=3.630 ; p<.001)$, y realizar búsquedas de información que les interesa $(M=2.92, D T=1.9 ; t=7.399 ; p<.001)$. También existen diferencias de género en los usos que hacen de los ordenadores en las siguientes actividades: procesadores de texto (mujeres, $M=2,51$ y hombres, $M=1.86, \quad \mathrm{t}=6.157 ; \quad p<.001)$, presentaciones digitales (mujeres, $M=2,25$ y hombres, $M=1.75$, t=4.895; $p<.001$ ) $\mathrm{y}$ ediciones de fotos (mujeres, $M=2.14 \mathrm{y}$ hombres, $M=1.44, \mathrm{t}=5.837 ; \mathrm{p}<.001)$ más frecuentes en mujeres, $\mathrm{y}$ hacer cálculos y procesar datos (mujeres, $M=.85$ y hombres, $M=.60, \mathrm{t}=-2.813 ; \mathrm{p}<.01)$ más frecuentes en hombres.

Tabla 1 .

Medias, desviaciones típicas y diferencias de género en el uso de internet y de los ordenadores.

\begin{tabular}{|c|c|c|c|c|c|}
\hline Uso de Internet y Ordenador & $\begin{array}{c}\text { Total } \\
\mathrm{N}=351 \\
M(D T)\end{array}$ & $\begin{array}{l}\text { Mujeres } \\
\mathrm{N}=190 \\
M(D T) \\
\end{array}$ & $\begin{array}{c}\text { Hombres } \\
\mathrm{N}=161 \\
M(D T) \\
\end{array}$ & $\begin{array}{c}t \\
(\mathrm{gl}=349)\end{array}$ & $p$ \\
\hline \multicolumn{6}{|l|}{ Uso de Internet } \\
\hline Escuchar música & $3,21(1,0)$ & $3.30(0.9)$ & $3.11(1.08)$ & 1.794 & .07 \\
\hline Ver videos/películas/series & $2,70(1.0)$ & $2.76(1.07)$ & $2.63(1.08)$ & 1.127 & .26 \\
\hline Juegos online & $1,50(1.3)$ & $0.94(1.04)$ & $2.16(1.36)$ & -9.282 & .001 \\
\hline Conectarse a redes sociales & $2,82(1.2)$ & $3.05(1.19)$ & $2.55(1.21)$ & 3.888 & .001 \\
\hline Chatear & $2,60(1.31)$ & $2.77(1.27)$ & $2.40(1.32)$ & 2.701 & .01 \\
\hline Enviar/leer emails & $1,85(1.04)$ & $2.04(1.04)$ & $0.70(.84)$ & 3.630 & .001 \\
\hline Participar en foros & $0,59(.82)$ & $0.49(.78)$ & $0.70(.83)$ & -2.303 & .05 \\
\hline Colaborar en Blogs, wikis, ... & $0,741.0)$ & $0.77(1.02)$ & $0.70(.83)$ & 731 & .46 \\
\hline Comprar o vender por internet & $0.92(1.0)$ & $0.83(1)$ & $1.021)$ & -1.755 & .08 \\
\hline Descargar música, películas o series & $2.43(1.1)$ & $2.47(1.11)$ & $2.38(1.17)$ & .775 & .43 \\
\hline Descargar programas para mi ordenador & $1.77(1.08)$ & $1.63(1)$ & $1.94(1)$ & -1.723 & .01 \\
\hline Buscar información que me interesa & $2.80(.99)$ & $2.92(1.9)$ & $2.67(.9)$ & 2.316 & .05 \\
\hline Navegar para matar el tiempo & $1.70(1.27)$ & $1.72(1.2)$ & $1.68(1.12)$ & .227 & .78 \\
\hline Consultar periódicos o webs de noticias & $1.54(1,14)$ & $1.48(1)$ & $1.61(1)$ & -1.049 & .29 \\
\hline Hacer trabajos o actividades de clase & $2.35(1.0)$ & $2.69(0.8)$ & $1.95(0.9)$ & 7.399 & .001 \\
\hline \multicolumn{6}{|l|}{ Uso del ordenador } \\
\hline Procesador de texto & $2.21(1)$ & $2.51(1)$ & $1.86(.9)$ & 6.157 & .001 \\
\hline Hacer presentaciones & $2.02(.9)$ & $2.25(1)$ & $1.75(.8)$ & 4.895 & .001 \\
\hline Hacer gráficos & $0.94(1.8)$ & $0.93(.9)$ & $0.95(.8)$ & -.255 & .79 \\
\hline Edición de fotos & $1.82(1.1)$ & $2.14(1)$ & $1.44(1)$ & 5.837 & .001 \\
\hline Edición de video o audio & $1.38(1.1)$ & $1.39(1.2)$ & $1.37(1.1)$ & .132 & .89 \\
\hline Procesar datos (hojas de cálculo) & $0.72(.8)$ & $0.60(.8)$ & 0.85 & -2.813 & .01 \\
\hline Bases de datos & $0.63(.8)$ & $0.58(.88)$ & $0.68(.84)$ & -1.125 & .26 \\
\hline Crear páginas web & $0.32(.7)$ & $0.28(.68)$ & $0.36(.76)$ & -.984 & .32 \\
\hline
\end{tabular}

Prueba constraste: Prueba t de Student para dos muestras independientes

En el cuestionario también se les preguntaba a los estudiantes sobre sus percepciones de autoeficacia, actitudes y creencias en estereotipos de género. La tabla 2 muestra las puntuaciones medias en las escalas que medían esas variables. En relación a la percepción de autoeficacia, tanto mujeres como hombres obtienen puntuaciones medias altas en la escala SEICT (mujeres $M=5,45, D T=.84$ y hombres $M=5.35, D T=1.01) \mathrm{La}$ percepción de autoeficacia es más baja para las tareas referidas al dominio de los computadores $(M=4.75 \mathrm{y}$ $M=4.74$ mujeres y hombres respectivamente) que para las referidas al dominio de internet (mujeres $M=6.40$, $\mathrm{DT}=.88$ y hombres $M=6.15, \mathrm{DT}=1.02$ ) en las cuales las mujeres manifiestan una mayor autoeficacia que los hombres $(t=2.339, \quad p<.05)$. No hay diferencias significativas de género en las actitudes hacia la informática, donde en base a las puntuaciones al CAS ambos sexos expresan actitudes moderadamente positivas. En cambio, los hombres puntúan más alto en la escala que mide los estereotipos de género, siendo las diferencias con las chicas estadísticamente significativas (mujeres $M=1.71, D T=.88$ y hombres 
$M=2.16, D T=1 ; \mathrm{t}=-4.253, p<.001)$.

Tabla 2.

Medias, desviaciones típicas y diferencias de género en las puntuaciones de las escalas que miden autoeficacia tareas de computadores, autoeficacia tareas de internet, actitudes, y estereotipos de género.

\begin{tabular}{lccccc}
\multicolumn{1}{c}{ Escalas } & Total & Mujeres & Hombres & $t$ & $p$ \\
& $\mathrm{~N}=351$ & $\mathrm{~N}=190$ & $\mathrm{~N}=161$ & $M(D T)$ & \\
& $M(D T)$ & $M(D T)$ & $5.35(1.01)$ & 1.046 & .289 \\
Self Efficacy Internet Computer Task (SEICT) & $5.40(.92)$ & $5.45(.84)$ & $4.74(1.28)$ & .030 & .976 \\
Subescala self-efficacy computer (SECT) & $4.74(1.17)$ & $4.75(1.08)$ & $6.15(1.02)$ & 2.339 & $\mathbf{. 0 2 0}$ \\
Subescala self-efficacy internet (SEIT) & $6.29(.95)$ & $6.40(.88)$ & $3.45(.45)$ & -.897 & .371 \\
Computer attitude Scale (CAS) & $3.43(.41)$ & $3.41(.37)$ & $2.16(1.08)$ & -4.253 & $\mathbf{. 0 0 1}$ \\
Estereotipo de rol sexual $(E E R S)$ & $1.92(1.00)$ & $1.71(.88)$ & &
\end{tabular}

Prueba constraste: Prueba t de student para dos muestras independientes

Finalmente, en la tabla 3 se muestran las relaciones entre la edad, la frecuencia de uso del ordenador, la percepción de autoeficacia, las actitudes hacia los ordenadores y las creencias en los estereotipos de género. Los resultados indican que hay relaciones significativas y positivas entre la frecuencia de uso de los ordenadores, las actitudes hacia los mismos $(r=.25$, $p<.001)$ y la percepción de autoeficacia $(r=.21, p<.001)$ tanto para las tareas computacionales $(r=.20, p<.001)$ como para las tareas con internet $(r=.14, \mathrm{p}<.01)$, de modo que cuanto más tiempo pasan usando el ordenador y conectados a internet, más positivas son las actitudes hacia ellos, y mayor es su percepción de autoeficacia. Por otra parte, autoeficacia y actitudes también están muy relacionadas, y a mayor puntuación en autoeficacia computacional y de internet, las actitudes hacia la informática son más positivas $(r=.29$, $p<.001)$. La edad no tiene una relación significativa con la frecuencia de uso pero si con la autoeficacia $(r=.17, p<.01)$, especialmente con la autoeficacia en internet $(r=.22, p<.001)$, con la actitudes $(r=.15, p<.01)$ y negativamente con los estereotipos de género $(r=.19$, $p<.001)$. Los estudiantes con más estereotipos de género respecto al dominio de los computadores son los que tienen menos edad ( $r=-19, p<.001)$, actitudes menos positivas sobre los computadores $(r=-21$, $p<.001)$, y menor percepción de autoeficacia informática, especialmente en la que se refiere al dominio de internet $(r=-.21, p<.001)$.

Tabla 3.

Intercorrelaciones entre edad, frecuencia de uso de ordenadores y las escalas que miden autoeficacia tareas de computadores, autoeficacia tareas de internet, actitudes, y estereotipos de género. Prueba de contraste: coeficiente correlación r de Pearson

\begin{tabular}{lcccccc}
\hline Variables & Edad & Frecuencia uso & SEICT & SECT & SEIT & CAS \\
\hline Edad & 1 & & & & & \\
Frecuencia uso & .09 & 1 & & & & \\
SEICT & $.17^{*}$ & $.21^{* *}$ & 1 & & & \\
SECT & .10 & $.20^{* *}$ & $.92^{* *}$ & 1 & 1 & 1 \\
SEIT & $.22^{* *}$ & $.14^{*}$ & $.76^{* *}$ & $.44^{* *}$ & $.34^{* *}$ & $-.21^{* *}$ \\
CAS & $.15^{* *}$ & $.25^{* *}$ & $.29^{* *}$ & $.19^{* *}$ & 1 \\
EERS & $-.19^{* *}$ & -.01 & -.10 & -.01 & $-.21^{* *}$ & 1
\end{tabular}

$* \mathrm{p}<.01 . * * \mathrm{p}<.001$

\section{Discusión}

En este trabajo pretendíamos analizar las diferencias de género entorno al dominio de las TICs entre los adolescentes. Nuestros resultados muestran que la llamada brecha de género, en lo que a su uso y dominio se refiere está cerrada. La inmensa mayoría de los jóvenes de nuestro estudio dispone de un ordenador y de otros dispositivos con acceso a internet, se pueden conectar desde casa y lo hacen a diario. Además llevan más de 5 años manejando un ordenador y accediendo a internet. Respecto a como han aprendido sus habilidades informáticas, suelen haberse formado por diversas fuentes. Principalmente aprenden de forma autodidacta, asistiendo a cursos y con el apoyo de sus padres. También, por lo que respecta a la percepción de autoeficacia y a las actitudes hacia los computadores e internet, las diferencias de género han desaparecido.
Las diferencias más claras entre chicos y chicas se dan en las actividades que realizan. Las mujeres, en comparación con los hombres, hacen un uso más frecuente de estas tecnologías para actividades relacionadas con el trabajo escolar, mientras que no se encuentran diferencias en la frecuencia en que realizan actividades de ocio. Si nos centramos en actividades más especificas en el uso de los ordenadores, las chicas utilizan, más a menudo que los chicos, software de presentaciones digitales y procesadores de texto (vinculados a sus tareas escolares), y edición de fotos. Por su parte, los chicos, en mayor medida que las mujeres, los usan más a menudo para procesar datos (hojas de cálculo) si bien no es una actividad frecuente. $\mathrm{Si}$ nos referimos a las actividades que realizan cuando se conectan a internet, las mujeres lo hacen con más frecuencia para buscar información que les interesa, 
conectarse a redes sociales, chatear o enviar emails, y los hombres para juegos online, descargar programas y participar en foros. Estas diferencias en los usos de internet y de los ordenadores, que ya se manifestaban en los estudios realizados hace más de una década (Acosta et al, 2003; Beierlein et al, 2006; Durndell y Haag, 2002), parecen seguir manteniéndose, como muestra el estudio de Joiner et al. (2012) realizado con universitarios en Gran Bretaña, cuyos resultados también indican que las mujeres se conectan más a redes sociales y los hombres a juegos online. La diferenciación entre hombres y mujeres en el uso que hacen de estas tecnologías parece evidente. Las mujeres parecen verlas como una herramienta de trabajo y comunicación y los hombres como un objeto de entretenimiento.

Por último y en relación al estereotipo de género en relación con la informática, aunque los jóvenes de nuestro estudio muestran, en general, su desacuerdo con esas creencias, este desacuerdo es menos pronunciado en los varones, presentando algunos de ellos una visión de la informática como parte de un domino más masculino que femenino. Los estereotipos son más comunes en sujetos con baja percepción de autoeficacia y actitudes negativas hacia los computadores e internet, y según nuestros datos, estas creencias disminuyen con la edad.

\section{Referencias}

Acosta, M., Borges, M., González, E., Hernández, C. y Rodríguez, E. (2003). Use of the ICTs and the Perception of E-learning among University Students: a Differential Perspective according to Gender and Degree Year Group. Interactive Educational Multimedia, 7, 13-28.

Beierlein, C., Imhof, M. y Vollmeyer, R. (2006). Computer use and the gender gap: The issue of access, use, motivation, and performance. Computers in Human Behavior, 23, 2823-2837

Durndell, A. y Haag, Z. (2002). Computer self efficacy, computer anxiety, attitudes towards the internet and reported experience with the internet, by gender, in an East European sample. Computers in Human Behavior, 18, 521-535.

Glassman, M. y Kim, Y. (2013). Beyond search and communication: Development and validation of the internet Self-efficacy Scale (ISS) Computers in Human Behaviour, 29, 1421-1429.

Hergatt, A., Whetten, J. y Huffman, W. (2013). Using technology in higher education: The influence of gender roles on technology self-efficacy. Computers in Human Behavior, 29, 1779-1786.

Joiner, R., Gavin, J., Brosnan, M., Cromby, J., Gregory, H., Guiller, J., Maras, P. y Moon, A. (2012). Gender, internet experience, internet identification and internet anxiety: a ten year follow up. Cyberpsychology, behavior and social networking, 15, 7, 370-372.

Li, N. y Kirkup, G. (2007) Gender and cultural differences in internet use: A study of China and the UK. Computers \& Education, 48, 301-317.
Li, Y. y Ranieri, M. (2013). Educational and social correlates of the digital divide for rural and urban children: A study on primary school students in a provincial city of China. Computers \& Education, 60, 197-209.

Nickell, G.S. y Pinto, J.N. (1986). The computer attitude scale. Computers in Human Behavior, 2, 301-306.

Papastergiou, M. (2008). Are computer science and information technology still masculine fields? High school students' perceptions and career choices. Computers and Education, 51(2), 594-608.

Simsek, A. (2011) The relationship between Computer Anxiety and Computer Self-Efficacy. Contemporary Educational Technology 2 (3), 177-187.

Tomte, C. y Hatlevik, O.E. (2011) Gender-differences in self-efficacy ICT related to various ICT-user profiles in Finland and Norway. How do selfefficacy, gender and ICT-user profiles relate to findings from PISA 2006. Computers \& Education, 57, 1416-1424.

Tsai, M-J. \& Tsai, C-C. (2010) Junior high school students' internet usage and self-efficacy: A reexamination of the gender gap. Computers \& Education, 54, 1182-1192

Vekiri, I. y Chronaki, A. (2008). Gender issues in technology use: Perceived social support, computer self-efficacy and value beliefs, and computer use beyond school. Computers \& Education, 51, 1392 1404.

Whitley, B. (1997). Gender Differences in ComputerRelated Attitudes and Behavior: A Meta-Analysis. Computers and Human Behavior, 13 (1), 1-22. 